\title{
SAN CESARIO DI ARLES E I SUOI CONTINUATORI E IL MONACHESIMO FEMMINILE
}

L'articolo intende presentare gli inizi del movimento monastico femminile in Gallia e, in seno ad esso, di quello propagato da san Cesario di Arles e dai suoi immediati continuatori, specialmente da sant'Aureliano di Arles. Sarà, perciò, preso in considerazione il periodo che si estende dall'inizio alla metà del secolo VI.

\section{MONACHESIMO FEMMINILE DI SAN CESARIO DI ARLES}

Le fonti per conoscere sia la vita di san Cesario che la sua attività monastica di stampo femminile sono le seguenti: 1) la Vita Sancti Caesarii ${ }^{1}$, scritta da più persone negli anni successivi alla morte di san Cesario: tre vescovi (il più importante di loro fu Cipriano di Tolone, discepolo di san Cesario, da lui ordinato vescovo di Tolone, prima del $515^{2}$ ), un sacerdote e un diacono; 2) alcuni documenti ufficiali del suo ministero episcopale: i concili gallici di quel periodo da lui presieduti ${ }^{3}$ e la corrispondenza di san Cesario con i vescovi di Roma ${ }^{4}$.

* Prof. dr. hab. Bazyli Degórski, O.S.P.P.E. - professore di patrologia e di teologia dogmatica dell'età patristica presso la Pontificia Università S. Tommaso d'Aquino a Roma, e professore di patrologia e di antropologia patristica alla Pontificia Facoltà Teologica "Teresianum" a Roma; email: osppe.roma@gmail.com.

${ }^{1}$ La Vita Sancti Caesarii è pubblicata in: ASanc (mensis Augusti) VI 50-83; ed. B. Krusch in, Vitae Caesarii episcopi Arelatensis libri duo, MGH Scriptores rerum Merovingicarum III, Hannoverae 1896, 433-501; ed. E. Bona, Vita sancti Cesarii Episcopi Arelatensis, Supplementi di Lexis 16, Amsterdam 2002; ed. M.-J. Delage - M. Heijmans, Vie de Césaire d'Arles, SCh 536, Paris 2010.

${ }^{2}$ Cf. V. Saxer, Cipriano di Tolone, DPAC I 683; idem, Cipriano di Tolone, NDPAC I 10361037; C. Kasper, Cipriano di Tolone, in: Dizionario di letteratura cristiana antica, ed. S. Döpp W. Geerlings - C. Noce, Città del Vaticano - Roma 2006, 190.

${ }^{3}$ San Cesario di Arles presiedette i seguenti concili: Concilium Arelatense (6 VI 524), ed. C. de Clercq, CCL 148A, Turnholti 1963, 42-46; Concilium Carpentoratense (6 XI 527), CCL 148A, 47 52; Concilium Arausicanum (3 VII 529), CCL 148A, 53-76; Concilium Vasense (5 XI 529), CCL 148A, 77-81; Concilium Massiliense (26 V 533), CCL 148A, 84-97.

${ }^{4} \mathrm{Cf}$. la lettera di papa Felice IV, del 3 II 528, CCL 148A, 51-52; la lettera di papa Bonifacio II, del 25 I 531, CCL 148A, 66-69; la risposta di Cesario a papa Bonifacio II, CCL 148A, 69; la lettera di papa Giovanni II all'episcopato gallico (circa la causa di Contumelioso), del 7 IV, CCL 148A, 86; la lettera di papa Giovanni II al clero di Riez, CCL 148A, 86-86; la lettera di papa Giovanni II a san Cesario, del 7 IV 534, CCL 148A, 87-96; la lettera di papa Agapito I a san Cesario, del 18 VII 
Da codeste fonti apprendiamo che san Cesario nacque nel 470 nel territorio della città di Cabillon (Chalon-sur-Saône) e che proveniva probabilmente da una famiglia facoltosa ${ }^{5}$. Ciò nonostante, ebbe una formazione culturale piuttosto mediocre ${ }^{6}$. All'età di diciott'anni, chiese al suo vescovo Silvestro (484-526) di poter diventare chierico e così, per circa due anni, rimase nella gerarchia della Chiesa cittadina ${ }^{7}$. Era però particolarmente attirato dalla vita ascetico-monastica, con ammirazione anche per l'esempio di quella femminile. Forse sarebbe diventato diacono e, poi, presbitero della Chiesa di Chalon, se non avesse avuto occasione di conoscere il monachesimo egiziano ${ }^{8}$. Per tale ragione, nel 490-491, si recò a Marsiglia all'abbazia di San Vittore, fondata da san Giovanni Cassiano. L'abate voleva tenere san Cesario con sé, ma egli, avendo appreso che nell'isola di Lerino si era formata una comunità monastica così severa che sembrava inutile intraprendere il rischioso viaggio fino all'Egitto monastico, andò proprio a Lerino, ove fu accolto nel monastero dell'abate san Porcario ${ }^{9}$ e lì ricevette la veste monastica ${ }^{10}$.

A Lerino ebbe, oltre alla preparazione teologico-monastico-liturgica, una formazione culturale e intellettuale, basata anzitutto - per quanto riguarda l'esegesi biblica - su Origene e sant'Agostino; su sant'Ambrogio di Milano e sullo stesso sant'Agostino d'Ippona per i temi teologici; e - per quanto concerne la dottrina ascetico-monastica - su san Giovanni Cassiano, sullo stesso san Porcario e su Fausto di Riez ${ }^{11}$.

535, CCL 148A, 96-97. Cf. anche J. Limmer, Konzilien und Synoden im spätantiken Gallien von 314 bis 696 nach Christi Geburt, Teil 1: Chronologische Darstellung, Wissenschaft und Religion 10, Frankfurt am Main 2004, 190-208.

${ }^{5}$ Cf. Vita S. Caesarii 1, 5.

${ }^{6}$ Cf. I. Gobry, Storia del monachesimo, I, Roma 1991, 627; B. Degórski, Il primissimo monachesimo della Gallia, DPa 15 (2006) 49; idem, Il monachesimo latino nel Mediterraneo (secc. IV-VI): protagonisti e legislazione, Lublin 2006, 141-142.

${ }^{7}$ Cf. Vita S. Caesarii 1, 4.

${ }^{8}$ Cf. Gobry, Storia del monachesimo, I, p. 627; Degórski, Il primissimo monachesimo della Gallia, p. 35; idem, Il monachesimo latino nel Mediterraneo, p. 125.

${ }^{9}$ Quinto abate di Lerino, governò probabilmente la comunità soltanto dal 485 al 489. Scrisse una specie di manuale della vita monastica, Monita, che è andato perduto. Cf. Degórski, Il monachesimo latino nel Mediterraneo, p. 113.

${ }^{10}$ Cf. Vita S. Caesarii $1,5$.

${ }^{11}$ Terzo abate di Lerino (dal 433 al 457), Fausto nacque nella Britannia poco prima del 410. Probabilmente verso il 424, si fece monaco a Lerino sotto l'abbaziato di sant'Onorato. Quando, nel 433, san Massimo, successore di sant'Onorato, divenne vescovo di Riez, Fausto gli succedette nell'abbaziato. Ordinato presbitero, si sforzò di mantenere il fervore primitivo della comunità. Intorno al 460, quando san Massimo morì, Fausto divenne vescovo di Riez, fino all'anno 477, quando il re dei Goti, Eurico, lo esiliò a causa del suo antiarianesimo e delle vicende collegate all'occupazione, da parte dei Visigoti, dell'Alvernia e della Provenza. Nel 462, Fausto partecipò ad un concilio romano in qualità di rappresentante dei vescovi della Gallia. Quindi, tornò a Riez probabilmente verso il 485. Per quando riguarda la data della sua morte, essa viene stabilita in base alla lettera di Avito (cf. Epistula 4 [Ad Gundobadum]), scritta prima del 500, dalla quale risulta che il vescovo di Riez 
L'abate Porcario lo costrinse, prima del 499, ad andare ad Arles, ove venne accolto da una coppia di pii laici: Gregoria e Firmino ${ }^{12}$.

Gregoria era parente dello stesso san Cesario. La casa di Gregoria e di Firmino era, a un tempo, un centro culturale, un ricovero, una mensa dei poveri, un ospedale, una sede di catechesi. In quella casa, ad Arles san Cesario frequentò le lezioni del retore africano Giuliano Pomerio ${ }^{13}$, che era allora il più celebre retore di Provenza, e si avvicinò, in tal modo, ai pensatori profani e alla filosofia. Durante un sogno, a san Cesario era apparso un serpente che lo divorava ${ }^{14}$, e così egli si convinse del pericolo causato dagli studi profani e abbandonò del tutto la cultura classica ${ }^{15}$.

Il vescovo di Arles, Eonio (o "Eone"; parente di san Cesario) ottenne dall'abate Porcario l'autorizzazione ad accogliere san Cesario come membro del suo clero ${ }^{16}$.

Nel 499 (o nel 500 ${ }^{17}$ ), a san Cesario fu affidata anche la direzione amministrativa di un monastero in suburbana insula civitatis $^{18}$ (probabilmente un quartiere sulla riva destra del fiume Rhône, l'odierna Trinquetaille $\left.{ }^{19}\right)^{20}$.

Dopo più di tre anni di assoluto isolamento, il vescovo Eonio ritenne di aver trovato in san Cesario il suo successore ${ }^{21}$. Cesario, però, fuggì per essere, poi, ritrovato nella necropoli di Alyscamps, una imponente area edificata sotto i bastioni del grande san Trofimo ${ }^{22}$. E soltanto nel 502 (o 503 23 ) egli divenne vescovo di Arles ${ }^{24}$. La città si trovava allora sotto il governo del re visigoto Alarico II che era ariano, e il vescovo Cesario dovette sopportare tante situazioni politiche difficili, dalle quali, però, seppe trarre vantaggi per la Chiesa cattolica ${ }^{25}$.

era deceduto già da alcuni anni. Fausto viene comunemente considerato esponente del semipelagianesimo. Cf. T. Sardella, Cesario di Arles, in: Quasten III 290.

${ }^{12}$ Cf. Gobry, Storia del monachesimo, I, p. 628; Sardella, Cesario di Arles, p. 290; Degórski, Il primissimo monachesimo della Gallia, p. 36; idem, Il monachesimo latino nel Mediterraneo, p. 126.

${ }^{13}$ A proposito di Giuliano Pomerio, cf. ad esempio: C. Tibiletti, La teologia della grazia in Giuliano Pomerio, "Augustinianum" 25 (1985) 489-506; M. Spinelli, Introduzione, in: Giuliano Pomerio, La vita contemplativa, Collana di Testi Patristici 64, Roma 1987, 7-41.

${ }^{14}$ Cf. Vita S. Caesarii 1, 9.

${ }^{15}$ Cf. Sardella, Cesario di Arles, p. 290-291; Degórski, Il primissimo monachesimo della Gallia, p. 36; idem, Il monachesimo latino nel Mediterraneo, p. 126.

${ }^{16}$ Cf. Gobry, Storia del monachesimo, I, p. 628-629; Sardella, Cesario di Arles, p. 291; Degórski, Il primissimo monachesimo della Gallia, p. 36; idem, Il monachesimo latino nel Mediterraneo, p. 126.

${ }^{17}$ Cf. Gobry, Storia del monachesimo, I, p. 629.

${ }^{18}$ Cf. Vita S. Caesarii 1, 12.

${ }^{19}$ Cf. Sardella, Cesario di Arles, p. 291.

${ }^{20}$ Cf. Gobry, Storia del monachesimo, I, p. 629.

${ }^{21}$ Cf. Sardella, Cesario di Arles, p. 291.

${ }^{22}$ Cf. Gobry, Storia del monachesimo, I, p. 631.

${ }^{23}$ Cf. ibidem, p. 629.

${ }^{24}$ Cf. Sardella, Cesario di Arles, p. 291.

${ }^{25}$ Cf. Gobry, Storia del monachesimo, I, p. 630; Sardella, Cesario di Arles, p. 291; Degórski, Il primissimo monachesimo della Gallia, p. 37; idem, Il monachesimo latino nel Mediterraneo, p. 128. 
Nonostante la vita esemplare e santa, il vescovo di Arles fu sospettato di tradimento ed esiliato a Burdigala, in residenza obbligatoria ${ }^{26}$. Ciò avvenne ad opera di un tale Liciniano che addirittura faceva parte del clero della diocesi di Arles. Infatti, nel 505, questo disgraziato chierico fece capire al re Alarico II che san Cesario cospirava contro lo stato insieme al re dei Burgundi, Gondebaudo. Così il vescovo di Arles fu arrestato ed esiliato. Ne approfittò per menare una vita eremitica. Nel 506, comunque, egli riconquistò la fiducia del re ${ }^{27}$.

Dimorando a Burdigala, san Cesario strinse rapporti con i vescovi della Gallia occidentale (Cipriano di Burdigala e Ruricio di Limoges). Sempre nel 506, il vescovo di Arles cominciò la costruzione del monastero femminile di San Giovanni ${ }^{28}$ che fu portata a termine nel $512^{29}$. Voleva che questo cenobio fosse situato vicino a lui per poterlo istruire e proteggere. Tuttavia, desiderava che sorgesse fuori della città di Arles, e ciò ovviamente per la tranquillità e il silenzio delle monache. Scelse, perciò, gli Alyscamps. L'opera gli stava tanto a cuore che vi partecipò egli stesso. Dopo la battaglia di Vouillé, però, in cui il re Clodoveo sconfisse Alarico II, Franchi e Burgundi misero l'assedio proprio davanti alla città di Arles e demolirono il monastero ormai quasi finito, per costruirne delle trincee. Gli Ostrogoti, poi, attaccarono gli assedianti, che dovettero ritirarsi. I costruttori, allora, ripresero la costruzione del monastero, che durò dal 508 al 513, data della sua consacrazione. La prima badessa di quel monastero fu santa Cesaria, sorella di san Cesario. Numerose vergini e vedove si unirono a lei. San Cesario diede loro una regola, e papa Ormisda approvò la fondazione del monastero con una bolla del $514^{30}$.

Alcuni mesi dopo la consacrazione del monastero, santa Cesaria morì e fu sostituita da un'altra Cesaria, chiamata la Giovane. Il numero delle monache salì allora a duecento. Alla morte di quell'ultima badessa, le successe santa Liliola e, quindi, santa Rusticola. Quest'ultima era figlia di una nobile donna di Vaison, Clemenza, che aveva perso il marito e il primogenito dei suoi due figli. In precedenza, Rusticola era assai bella, anche se aveva soltanto all'epoca cinque anni, e un cavaliere burgundo, Cheraone, decise di rapirla, volendo sposarla. Avvertita di ciò dalla madre, Liliola scrisse al vescovo Siagrio d'Autun e al re Gontrano per ricevere giustizia. Così, il guerriero rapitore dovette restituire Rusticola, che fu data a Liliola. Alla bambina piacque quella vita monastica austera e penitenziale: andava con le religiose agli uffici liturgici e aveva imparato a memoria tutto il salterio e gran parte della Sacra Scrittura. Rusticola era,

${ }^{26}$ Cf. Vita S. Caesarii 1, 21.

${ }^{27}$ Cf. Gobry, Storia del monachesimo, I, p. 630; Degórski, Il primissimo monachesimo della Gallia, p. 38; idem, Il monachesimo latino nel Mediterraneo, p. 128.

${ }^{28} \mathrm{Cf}$. C. Lambot, Le prototype des monastères cloîtrés de femmes: l'Abbaye Saint-Jean d'Arles (VIe siècle), "Revue Liturgique et Monastique" 23 (1938) 169-174.

${ }^{29}$ Cf. Sardella, Cesario di Arles, p. 291.

${ }^{30}$ Cf. Gobry, Storia del monachesimo, I, p. 631; Degórski, Il primissimo monachesimo della Gallia, p. 38; idem, Il monachesimo latino nel Mediterraneo, p. 129. 
inoltre, una monaca esemplare e virtuosa. Quando chiese di diventare monaca e di indossare l'abito monastico, la badessa acconsentì. La madre di lei, però, ne fu adirata e cercò di far cambiare il proposito della figlia con la bella villa e le ricchezze di Vaison. La causa fu allora affidata al vescovo Sapaudo. Secondo la legge, una vergine che aveva passato parecchi anni in un monastero non poteva essere mandata via contro la sua volontà. La madre Clemenza si ricordò, allora, di un sogno: san Cesario la vide nella sua casa con due colombe sulle ginocchia: ne aveva reclamata una; Clemenza gliel'aveva data ed egli l'aveva portata via. Clemenza dovette, perciò, offrire al Signore la sua amata figlia. Dopo la morte di Liliola, Rusticola (all'età di diciotto anni) fu eletta badessa ${ }^{31}$.

Da quando la regione passò alla dominazione degli Ostrogoti (508-536), san Cesario di nuovo fu accusato di tradimento e fu convocato a Ravenna dal re Teoderico $(512-513)^{32}$, ma riuscì a dimostrare la propria innocenza e ottenne la liberazione della popolazione di Orange, che era stata fatta prigioniera ${ }^{33}$. San Cesario si recò, quindi, da Ravenna a Roma ed incontrò papa Simmaco dal quale ottenne, nel 513, la conferma dei diritti metropoliti della Chiesa di Arles contro quella di Vienne (allora diretta dal vescovo Avito) ${ }^{34}$.

Nel 514, papa Simmaco confermò a san Cesario i privilegi di vicario della Sede Apostolica per la Spagna e per la Gallia. Da quell'anno, quindi, san Cesario esercitò un influsso sempre più crescente su tutta la Gallia ${ }^{35}$.

Nel 534, san Cesario ultimò definitivamente la regola delle monache, e nel 536 vide passare la città di Arles sotto il governo dei cattolici, con il re franco Childeberto $\mathrm{I}^{36}$.

Nell'ultimo anno della vita (543), la salute di san Cesario diminuì sempre di più. Aspettava per morire la festa di sant'Agostino. Quando questo giorno

${ }^{31}$ Cf. Gobry, Storia del monachesimo, I, p. 632; C. Thiellet, La Règle de saint Césaire d'Arles et les fondations monastiques féminines, in: Pratique et sacré dans les espaces monastiques au MoyenÂge et à l'époque moderne. Actes du colloque de Liessies- Maubeuge (26-28 septembre 1997), éd. R.-H. Bautier - Ph. Racinet, Amiens 1998, 23-33; Degórski, Il primissimo monachesimo della Gallia, p. 39; idem, Il monachesimo latino nel Mediterraneo, p. 130.

${ }^{32}$ Circa Teoderico il Grande, cf. ad esempio: W. Ensslin, Theoderich der Grosse, München 1947; P. Lamma, Teodorico, La Scuola Editrice, Brescia 1951; J. Moorhead, Theoderic in Italy, Oxford 1992; P. Amory, People and identity in Ostrogothic Italy, 489-554, Cambridge 1997; A. Giovanditto, Teodorico e i suoi Goti in Italia (454-526), Milano 1998; B. Saitta, La "civilitas" di Teodorico: rigore amministrativo, "tolleranza" religiosa e recupero dell'antico nell'Italia ostrogota, Roma 1999.

${ }^{33}$ Cf. Vita S. Caesarii 1, 38. Cf. anche Sardella, Cesario di Arles, p. 291.

${ }^{34}$ Cf. Gobry, Storia del monachesimo, I, p. 633; Degórski, Il primissimo monachesimo della Gallia, p. 39; idem, Il monachesimo latino nel Mediterraneo, p. 130.

${ }^{35}$ Cf. Concilium Arelatense (6 VI 524), CCL 148A, 42-46; Concilium Arausicanum (3 VII 529), CCL 148A, 53-76.

${ }^{36}$ Cf. Degórski, Il primissimo monachesimo della Gallia, p. 5-66. Per quanto riguarda Childeberto I, cf. ad esempio: Ch. Pfister, La Gallia sotto i Franchi merovingi, vicende storiche, in: Storia del mondo medievale, I, Milano 1999, 688-711; ibidem, La Gallia sotto i Franchi merovingi, istituzioni, in: Storia del mondo medievale, I, Milano 1999, 712-742; R. Altamira, La Spagna sotto $i$ visigoti, in Storia del mondo medievale, I, Milano 19997, 743-779. 
si avvicinò, egli volle essere trasportato all'ora dell'ufficio liturgico presso le monache di San Giovanni. Esse furono molto commosse. San Cesario si congedò per sempre dalla badessa Cesaria e rivolse alle monache l'esortazione ad essere fedeli alla regola monastica. Impartì, quindi, a tutte la sua benedizione. Vicino al letto del moribondo si riunirono alcuni vescovi della sua provincia ecclesiastica, i presbiteri, i diaconi, gli amici. San Cesario morì proprio il 27 agosto 543, la vigilia di sant'Agostino d'Ippona, nel settantaquattresimo anno di età e nel quarantunesimo di episcopato. Il suo corpo fu tumulato nella basilica di Santa Maria ${ }^{37}$.

Vediamo ora l'operato di san Cesario di Arles. Anzitutto, egli fu un vero e proprio pastore d'anime, fondatore di istituzioni di carità e di chiese, legislatore liturgico e monastico della sua regione ecclesiastica: compose due regole monastiche, una per monaci ${ }^{38}$ e una per monache, e fondò diversi monasteri ${ }^{39}$.

Il metodo di lavoro di san Cesario, che si serviva degli estratti di vari autori, prevalentemente di sant'Ambrogio di Milano e di sant'Agostino d'Ippona, aveva fatto sì che già alla fine del Medioevo la sua produzione letteraria fosse diventata anonima. Le sue opere furono recuperate soltanto alla fine dell'Ottocento dal famoso benedettino G. Morin ${ }^{40}$.

Gli Statuta sanctarum virginum ${ }^{41}$, cominciati verso il 512 (alla data della fondazione del monastero di San Giovanni), sono molto più ampi rispetto alle regole date da lui ai suoi monaci e dettagliati e, successivamente, furono più volte ritoccati, e quindi emendati, per consenso dello stesso san Cesario e così, nel 534, furono del tutto stilati. Questa regola è stata l'opera di un fondatore atta a prevenire ogni disordine e ad assicurare alla vita comunitaria solide e definitive fondamenta. La redazione di una regola concepita per delle monache fu, all'epoca, un fatto innovativo e suscitò interesse ed ammirazione (persino da parte di papa Ormisda, come testimonia la sua lettera indirizzata a san Cesario ${ }^{42}$ ). Tra le fonti di questa regola femminile troviamo le opere monastiche

${ }^{37}$ Cf. Vita S. Caesarii 2, 50. Cf. anche Gobry, Storia del monachesimo, I, p. 636; Degórski, Il primissimo monachesimo della Gallia, p. 41; idem, Il monachesimo latino nel Mediterraneo, p. 133.

${ }^{38}$ Cf. A. de Vogüé, La Règle de Saint Césaire d'Arles pour les moines: un résumé de sa Règle pour les moniales, RAM 47 (1971) 369-406.

${ }^{39}$ Cf. Sardella, Cesario di Arles, p. 292; Degórski, Il primissimo monachesimo della Gallia, p. 41; idem, Il monachesimo latino nel Mediterraneo, p. 133. Per quanto riguarda la bibliografia concernenti i testi monastici di san Cesario, cf. G. Terraneo, Saggio bibliografico su Cesario di Arles, SC 91 (1963) 232-294; J. Piłat, Św. Cezary z Arles. Bibliografia-aktualny stan badań, in: Oblicza doskonałości chrześcijańskiej, red. M. Chmielewski, Lublin 1996, 206-218.

${ }^{40}$ Cf. Sardella, Cesario di Arles, p. 292; Degórski, Il primissimo monachesimo della Gallia, p. 42; idem, Il monachesimo latino nel Mediterraneo, p. 133.

${ }^{41}$ In PL 67, 1105-1120; ed. G. Morin in: Sancti Caesarii episcopi Arelatensis, Opera omnia, I, Maredsous 1937, 100-127; éd. A. de Vogüé - J. Courreau in: Césaire d'Arles, Oeuvres Monastiques, SCh 345, Paris 1988, 170-272.

${ }^{42}$ Cf. Epistolae Romanorum Pontificum, ed. A. Thiel, I, Braunsberg 1868, 739ss. 
di san Giovanni Cassiano ${ }^{43}$, la tradizione ascetica dell'isola di Lerino, la regola maschile detta di sant' Agostino ${ }^{44}$. Ciò nonostante, bisogna anche sottolineare l'impronta originale dello stesso vescovo di Arles, sia per quanto riguarda le norme generali di comportamento (la stretta clausura, la vita comunitaria, l'importanza della lettura e della carità), sia nei diversi dettagli (come, ad esempio, 1'altezza dell'acconciatura delle monache) $)^{45}$.

Una simile regola per le monache era davvero necessaria. Infatti, i primissimi conventi femminili della Gallia meridionale somigliavano più a pensioni per nubili che non a luoghi di rinuncia e di contemplazione. Ogni monaca, per esempio, portava nella sua cella il proprio mobilio e le proprie abitudini ed usanze, i vestiti e l'arredamento del letto, persino la domestica e le ricette di cucina. Le monache non uscivano dalla clausura ma, in compenso, ricevevano frequentemente i visitatori che portavano a loro regali. Per questa ragione, dato che mancava proprio una vera regola monastica, san Cesario ne scrisse una, prima ancora di riunire le sue monache, e affidò la sorella alla comunità di Marsiglia, affinché imparasse la vita veramente monastica ${ }^{46}$.

Ora, vediamo più da vicino gli Statuta sanctarum virginum. Essi furono stilati per il monastero femminile di Arles. Ciò si deduce, ad esempio, dalle norme che stabiliscono la porta attraverso la quale le monache devono entrare ed usci$\mathrm{re}^{47}$. Questa regola femminile fu composta progressivamente e, perciò, contiene delle ripetizioni, aggiunte e dei cambiamenti non sempre in sintonia con delle altre norme. Le fonti della "Regola" sono prevalentemente la "Regola" agostiniana $^{48} \mathrm{e}$, secondariamente, gli scritti monastici di san Giovanni Cassiano ${ }^{49}$. La

${ }^{43}$ La Regola si ispira a san Giovanni Cassiano più che al suo maestro Agostino, e insiste sulla vita puramente comunitaria e sulla gerarchia delle cariche. La monaca deve impegnarsi alla stabilità. Il monastero "esente" dalla giurisdizione del vescovo è dipende dirette del papa. San Cesario perfezionò questa regola femminile fino alla sua morte.

${ }^{44}$ Cf. C. Lambot, La Règle de S. Augustin et S. Césaire, RBen 41 (1929) 331-341; L. de Seihac, Remarques sur l'utilisation par Césaire d'Arles de la "Regula Augustini, "Regulae Benedicti Studia. Annuarium internationale" 1 (1972) 287-291; idem, L'Utilisation par s. Césaire d'Arles de la Règle de s. Augustin. Étude de terminologie et doctrine monastique, Studia Anselmiana 62, Roma 1974.

${ }^{45}$ Cf. Sardella, Cesario di Arles, p. 292; Degórski, Il primissimo monachesimo della Gallia, p. 44; idem, Il monachesimo latino nel Mediterraneo, p. 136.

${ }^{46}$ Cf. Gobry, Storia del monachesimo, I, p. 641; Degórski, Il primissimo monachesimo della Gallia, p. 44; idem, Il monachesimo latino nel Mediterraneo, p. 136.

${ }^{47}$ Cf. gli articoli 59 e 73 .

${ }^{48}$ Il testo originale latino della "Regola" agostiniana è pubblicato in: PL 32, 1377-1384; ed. A. Goldbacher, CSEL 57, Vindobonae - Lipsiae 1911, 356-371; P. Schröder, Die Augustinerchorherrenregel: Entstehung, kritischer Text und Einführung der Regel, "Archiv für Urkundenforschung" 9 (1926) 271-306; D. de Bruyne, La première Règle de saint Benoît, RBen 42 (1930) 316-342; A.C. Vega, La Regla de San Agustín. Edición crítica, precedida de un estudio sobre la misma y los códices de El Escorial, El Escorial 1933; R. Arbesmann - W. Huempfner, Jordani de Saxonia liber vitae fratrum, New York 1943, 491-504; L. Verheijen, La Règle de saint Augustin, Collection des Études Augustiniennes, Paris 1967 (Obiurgatio: 105-107; Ordo monasterii: 148-152; Praeceptum: 417-437).

${ }^{49}$ Cf. Ioannes Cassianus, De institutis coenobiorum, PL 49, 43-476; ed. M. Petschenig, CSEL 
"Regola" comprende tre parti: la prima parte è la regola primitiva, data alle religiose all'inizio della fondazione del monastero di San Giovanni (ossia, verso il $\left.512^{50}\right)$. Questa prima parte contiene quarantasette articoli (1-47). La seconda parte seconda parte - chiamata Recapitulatio ${ }^{51}$ - concerne gli articoli 48-65 ed è stata aggiunta per risolvere diverse difficoltà pratiche ${ }^{52}$. E la terza parte, infine, chiamata Ordo psalmodiae e che abbraccia gli articoli 66-71, costituisce una parte liturgica della Regola. Nota bene, san Cesario inserì qui l'Ordo psalmodiae di Lerino (gli articoli 68-70), trapiantando in tal modo ai monasteri della Gallia meridionale le usanze liturgiche dei monaci lerinesi.

Analizziamo ora più da vicino la stessa "Regola" femminile di san Cesario.

1. Ammissione al monastero, clausura e stabilità. Al monastero potevano già essere ammesse persino bambine di 6-7 anni (art. 7). La candidata veniva provata per un anno intero abitando con una monaca, esperta della vita claustrale, che la introduceva alla prassi e alle regole del monastero. L'entrata nel monastero iniziava una conversio, un profondo cambiamento di vita della monaca (art. 3). In quel periodo, la candidata doveva molte volte leggere l'intera "Regola" e soltanto allora, superata questa prova, poteva essere ammessa alla vestizione monastica (art. 3 e 58) che all'epoca equivaleva ai voti religiosi dei nostri tempi. La base della vestizione era la compunctio, ossia la consapevolezza della propria peccaminosità e pochezza di fronte a Dio (art. 3). Le monache dovevano vivere nella povertà (art. 52) e dimenticare del tutto la loro condizione sociale precedente (art. 21). Per tale ragione, le suppellettili nel monastero dovevano essere semplici e modeste (art. 44).

Essendo separate dal mondo, le monache non potevano fare le madrine (art. 10). In più, erano tenute alla stabilitas loci rimanendo nello stesso monastero per tutta la vita (art. 1). Nel monastero non potevano entrare né donne, né uomini (art. 36-37), e soltanto eccezionalmente poteva accedervi un pio chierico, un vescovo o un artigiano (art. 39). Tali visite avvenivano in un luogo stabilito in presenza di una delle monache (art. 40). Inoltre, per essere separate dal mondo, le monache non potevano ricevere lettere (art. 25), né preparare pasti per nessuna persona estranea (art. 53 e 59), e dell'elemosina si occupava un provisor (art. 42). Egli si occupava anche dei beni del monastero e della sua amministrazione.

17, Pragae - Vindobonae - Lipsiae 1888, 3-231; J.-C. Guy, SCh 109, Paris 1965. Ioannes Cassianus, Conlationes patrum, PL 49, 477-1321; ed. M. Petschenig, CSEL 13a, Wien 2004; ed. E. Pichery, t. 1: I-VII, SCh 42, Paris 1955; t. 2: VIII-XVII, SCh 54, Paris 1958; t. 3: XVIII-XXIV, SCh 64, Paris 1959. Cf. anche A. de Vogüé, Une interpolation inspirée de Cassien dans un texte monastique Césaire d'Arles, SMon 25 (1983) 217-221.

${ }^{50}$ Secondo I. Gobry (Storia del monachesimo, I, p. 641), nel 513.

${ }^{51}$ In PL 67, 1115-1120.

${ }^{52}$ A proposito del contenuto degli Statuta sanctarum virginum, cf. anche Gobry, Storia del monachesimo, I, p. 642-646. 
2. La povertà. Prima di entrare nel monastero, la candidata doveva rinunciare, anche giuridicamente, a tutti i suoi averi, e dopo l'entrata ogni dono materiale doveva essere consegnato alla comunità (art. 43 e 54), quindi ogni monaca non doveva possedere niente (art. 52) e tanto meno lo doveva una inserviente (art. 17).

L'abito della religiosa doveva essere modesto, fatto nello stesso monastero, e modesta doveva essere la vita nello stesso monastero (art. 26, 44-45 e 55). Ciò nonostante, il monastero doveva essere abbastanza "ricco", perché ci vivevano circa duecento monache che filavano la lana che con tantissima probabilità proveniva dalle pecore del monastero, dato che la "Regola" non parla affatto di qualche acquisto di essa e, in compenso, parla degli schiavi che ivi lavoravano (art. 7) ${ }^{53}$.

3. La vita nel monastero. La vita nel monastero è pervasa dalla preghiera, specialmente dalla ripetizione privata dei versetti scritturistici (meditari) e recitando i Salmi le monache dovevano contemplarli con il cuore, perciò non dovevano fare alcun lavoro manuale durante l'ufficio delle ore (art. 10). Tuttavia, potevano lavorare durante l'ufficio notturno, affinché non dormissero (art. 15). Quelle che venivano in ritardo alla preghiera, erano punite (art. 12). Per poter pregare adeguatamente, le monache dovevano leggere due ore ogni giorno (art. 19), e per farlo dovevano ovviamente imparare a leggere (art. 18).

Nel monastero doveva regnare la concordia e l'unanimità. Le monache, perciò, dovevano essere ubbidienti (art. 13), non litigare, non mormorare, non picchiarsi, non insultarsi, ma stimarsi a vicenda (art. 26). Quindi, se una monaca era litigiosa, doveva fare penitenza; pena addirittura l'esclusione dalle preghiere comunitarie o dalla mensa comunitaria (art. 34).

Le monache dormivano in un dormitorio, in una sala comune (art. 8 e 29). Anche lavorano comunemente, conformemente agli ordini della superiora che indicava ad essere i lavori concreti da fare, anche se il loro lavoro principale consisteva nel fabbricare la lana e nel filarla (art. 16 e 57). Tutte le monache, eccetto la badessa e la priora, dovevano lavorare in cucina, per turnum ${ }^{54}$.

I digiuni delle monache erano meno severi rispetto a quelli dei monaci. Mentre mangiavano, le religiose dovevano osservare il silenzio ascoltando una lettura. La badessa, se non impedita, doveva mangiare insieme con la comunità. Le pietanze del monastero sono precisamente indicate dalla "Regola" (art. 71). La "Regola" si occupa parecchie volte anche delle monache malate e deboli (art. 31-32 e 42) 55 .

Va da sé che le monache dovevano vivere castamente, perché ciò si addice addirittura ad ogni cristiano come tale, e dovevano essere umili. Prima di

${ }^{53}$ Cf. M. Starowieyski, Prawodawstwo św. Cezarego z Arles, in: Zachodnie Reguly monastyczne, ŹM 50, Kraków - Tyniec 2013², 393.

${ }^{54}$ Cf. ibidem, p. 395.

${ }^{55} \mathrm{Cf}$. ibidem. 
denunciare alla superiora qualche comportamento sbagliato della consorella, bisognava ammonire privatamente colei che errava (art. 24). Per alcune colpe e trasgressioni la monaca poteva essere addirittura esclusa dalla comunità (art. 34).

4. La direzione del monastero. Il monastero veniva diretto da una badessa (abbatissa), chiamata anche "madre del monastero" (mater monasterii). Per esercitare tale ufficio doveva essere scelta la migliore delle monache (art. 61). Dato che la sua responsabilità davanti a Dio era grande, le consorelle dovevano ubbidirle (art. 18, 35).

La badessa si occupava di tutti i bisogni delle monache, stabiliva tutte le loro mansioni e i compiti e, inoltre, curava tutti gli affari e i contatti fuori del monastero ${ }^{56}$.

La badessa era responsabile per adempiere a tutte le norme stabilite dalla "Regola", la quale doveva essere scrupolosamente messa in atto. Però, se la badessa avesse voluto cambiare qualcosa che era richiesto dalla "Regola", le stesse monache, salva tutta la riverenza verso la badessa, avrebbero dovuto addirittura opporsene con tutte le loro forze (art. 65).

La badessa, per quanto riguarda la direzione del monastero, veniva aiutata dalla priora (praeposita, primiceria). Il termine, però, primiceria poteva indicare anche una delle suore più esperte, anziane (seniores) oppure la responsabile di una parte della comunità ${ }^{57}$. Un'altra aiutante della badessa era la portinaia (posticiaria; ad ad posticium). Ella, controllata dalla stessa badessa, manteneva le relazioni con gli estranei, con coloro che non appartenevano al monastero. La portinaia, però, non doveva trarne alcun vantaggio per se stessa. I monasteri gallici di quel periodo furono sottoposti alla guida e alla giurisdizione del vescovo del luogo. Il vescovo, quindi, poteva ingerire liberamente negli affari e nella vita spirituale degli stessi. Lo stesso san Cesiario volle esonerare i suoi monasteri dalla giurisdizione del vescovo del luogo, quindi lo aveva chiesto a papa Ormisda, il quale acconsentì alla domanda ${ }^{58}$.

Degli altri scritti monastici di san Cesario, possediamo anche i sei Sermones ad monachos e le Ad sanctimoniales epistulae (la Coegisti e la Vereor) ${ }^{59}$ indirizzate alla sorella Cesaria e alla sua comunità monastica, ossia al monastero femminile di Arles dedicato a San Giovanni ${ }^{60}$.

\footnotetext{
${ }^{56}$ Cf. ibidem, p. 393.

${ }^{57} \mathrm{Cf}$. G. Morin, Problèmes relatifs à la Règle de S. Césaire d'Arles pour les moniales, RBen 44 (1932) 5-20.

${ }^{58}$ Cf. Starowieyski, Prawodawstwo św. Cezarego z Arles, p. 394.

${ }^{59}$ Pubblicate in PL 67, 1128C - 1135C; ed. G. Morin, Sancti Caesarii episcopi Arelatensis, Opera omnia, II, Maredsous 1942, 134-144; éd. A. de Vogüé - J. Courreau, SCh 345, 294-336.

${ }^{60}$ Cf. Sardella, Cesario di Arles, p. 292-293; Degórski, Il primissimo monachesimo della Gallia, p. 44; idem, Il monachesimo latino nel Mediterraneo, p. 137.
} 
Esiste anche l'Epistula hortatoria ad virginem Deo dicatam ${ }^{61}$. G. Morin, però, non la considera scritta da san Cesario; essa dev'essere, forse, attribuita al nipote Teridio ${ }^{62}$.

\section{I CONTINUATORI DELL'OPERA MONASTICA FEMMINILE DI SAN CESARIO D'ARLES}

L'influsso di san Cesario sul monachesimo fu considerevole e durò per molte generazioni. Ciò nonostante, la sua Regula non ebbe tanta diffusione fuori della Provenza. Infatti, per quanto riguarda la Gallia meridionale, i cenobi che vivevano secondo essa scomparvero già un secolo dopo, e ciò a causa delle incursioni dei Saraceni. In seguito, dopo la liberazione, era già conosciuta ed apprezzata un'altra regola, quella di san Benedetto da Norcia ${ }^{63}$. Dobbiamo anche dire che la "Regola" di san Cesario e quella di san Benedetto hanno degli elementi comuni. Non possiamo, però affermare con esattezza se ci fosse stato un influsso dell'una sull'altra (ma quale su quale?), oppure se ambo le "Regole" avessero attinto da una fonte comune ${ }^{64}$.

Le comunità monastiche formate da san Cesario diedero alla luce personalità ecclesiastiche di alto rango. Il monastero di Saint-André di Villeneuve, vicino ad Avignone, ha un'origine poco chiara, ma una cronaca vi fa morire, nel 586, una certa santa Cesaria, monaca, che deve appartenere alla famiglia di san Cesario ${ }^{65}$.

Per quanto riguarda i primissimi monasteri femminili della Gallia meridionale, le notizie sono ancora più scarse. Possediamo, in proposito, una lettera di san Cesario di Arles indirizzata ad una badessa ${ }^{66}$. Da detto documento possiamo dedurre che ella fosse una sua figlia spirituale e che guidasse una comunità monastica di cui il vescovo di Arles era il fondatore ${ }^{67}$. Infine, il monastero di Saint-Pierre du Puy, ad Orange, che era benedettino nel XII secolo e cisterciense nel XIII, fu stabilito dallo stesso san Cesario sul monte Eutropio, prima di essere spostato all'interno della cinta muraria della città ${ }^{68}$.

\footnotetext{
${ }^{61}$ In PL 67, 1135-1138; éd. A. de Vogüé - J. Courreau, SCh 345, 418-438.

${ }^{62} \mathrm{Cf}$. G. Morin, Le prêtre arlésien Teridius, propagateur des Régles de S. Céaire d'Arles, RSR 27 (1938) 257-263; Sardella, Cesario di Arles, p. 293.

${ }^{63}$ Cf. Gobry, Storia del monachesimo, I, p. 646; Degórski, Il primissimo monachesimo della Gallia, p. 45; idem, Il monachesimo latino nel Mediterraneo, p. 137.

${ }^{64}$ Cf. Starowieyski, Prawodawstwo św. Cezarego z Arles, p. 398.

${ }^{65}$ Cf. Gobry, Storia del monachesimo, I, p. 648; Degórski, Il primissimo monachesimo della Gallia, p. 45; idem, Il monachesimo latino nel Mediterraneo, p. 137-138.

${ }^{66}$ I. Gobry (Storia del monachesimo, I, p. 649) la chiama: "Oratoria".

${ }^{67} \mathrm{Cf}$. Caesarius Arelatensis [?; Teridius ?], Epistula hortatoria ad virginem Deo dicatam, PL 67, 1135-1138; éd. A. de Vogüé - J. Courreau, SCh 345, 418-438.

${ }^{68} \mathrm{Cf}$. Gobry, Storia del monachesimo, I, p. 649; Degórski, Il primissimo monachesimo della Gallia, p. 49; idem, Il monachesimo latino nel Mediterraneo, p. 141-142.
} 
Durante il governo monastico della badessa santa Liliola, una donna si presentò al convento di San Giovanni, per essere iniziata alla "Regola" di san Cesario: era Radegonda, moglie separata di re Clotario, che, costretta a sposarsi, aveva infine ottenuto che le si rendesse la libertà, e che nel 415 aveva fondato a Poitiers il monastero di Santa Croce. Radegonda, cercando una regola che potesse assicurare (alle principesse e alle nobildonne che l'avevano seguita nel chiostro) sia la disciplina monastica che una certa moderazione, aveva sentito dell'equilibrio in cui vivevano le monache di Arles. Così, nel 568, Radegonda chiese a Liliola una copia della regola di san Cesario e, dopo averla letta, venne con la badessa Agnese ad Arles per viverla e apprenderla. In seguito, di ritorno a Poitiers, Radegonda e Agnese adottarono questa regola per la loro comunità ${ }^{69}$.

Un altro continuatore dell'opera monastica di san Cesario fu, nel VI secolo, sant'Aureliano († 16 giugno del 551), il secondo successore di san Cesario di Arles, dove rimase soltanto dal 546 alla morte. Ciò nonostante, in questo breve periodo, egli fondò ad Arles due monasteri: prima di San Pietro e San Paolo $(\text { maschile })^{70}$, nel $547^{71}$, a cui diede come abate Fiorentino, che aveva all'epoca sessantacinque anni e che sarebbe morto cinque anni dopo (nel 553) $)^{72} \mathrm{e}$, poi, di Santa Maria (femminile) $)^{73}$ che si trovava fuori le mura della città, in un luogo a noi sconosciuto. Ivi le monache recitavano l'ufficio divino in presenza dei fedeli che potevano accedere alla loro chiesa ${ }^{74}$.

Aureliano scrisse due nuove regole: la Regula ad monachos ${ }^{75}$ e la Regula ad virgines $^{76}$. Queste due opere sono le uniche che conosciamo di sant'Aureliano, e si ispirano prevalentemente a san Cesario di Arles $^{77}$ (specialmente attingono dalle sue Regulae, dai discorsi, dalla già menzionata lettera Vereor, e dalla Vita S. Caesarii $^{78}$ ) e, secondariamente, a sant'Agostino d'Ippona (l'Ordo monasterii e il Praeceptum) e a san Giovanni Cassiano (il De institutis coenobiorum). Inoltre, sant'Aureliano attinse anche dalle "Regole" di san Basilio, dalla "Seconda

${ }^{69}$ Cf. Venantius Fortunatus, Vita Sanctae Radegundis reginae. Cf. anche Gobry, Storia del monachesimo, I, p. 649; J. Pilvousek - K.B. Springer, Caesarius von Arles und die Klostergründung der heiligen Radegunde, in: Radegunde - ein Frauenschicksal zwischen Mord und Askese: eine Ausstellung, hrsg. H. Eidam - G. Noll, Erfurt 2006, 79-95.

${ }^{70}$ Cf. Y. Hen, Scrittori della Gallia, in: Quasten III 296; Degórski, Il primissimo monachesimo della Gallia, p. 45; idem, Il monachesimo latino nel Mediterraneo, p. 138.

${ }^{71}$ Cf. Starowieyski, Prawodawstwo św. Cezarego z Arles, p. 462.

${ }^{72}$ Cf. Gobry, Storia del monachesimo, I, p. 650.

${ }^{73}$ Cf. ibidem; Hen, Scrittori della Gallia, p. 296; Degórski, Il primissimo monachesimo della Gallia, p. 49; idem, Il monachesimo latino nel Mediterraneo, p. 142.

${ }^{74}$ Cf. Starowieyski, Prawodawstwo św. Cezarego z Arles, p. 462.

${ }^{75}$ La regola è pubblicata in PL 68, 385-398; A. Schmidt, Zur Komposition der Mönchsregel des heiligen Aurelian von Arles, SMon 17 (1975) 237-256 (edizione critica) e SMon 18 (1976) 17-54.

${ }^{76}$ La regola è pubblicata in PL 68, 399-406. Cf. anche A. de Vogüé, Regula(e) Aureliani, DIP VII 1604-1607.

${ }^{77}$ Cf. Gobry, Storia del monachesimo, I, p. 650; Hen, Scrittori della Gallia, p. 296.

${ }^{78}$ Cf. Starowieyski, Prawodawstwo św. Cezarego z Arles, p. 462. 
Regola dei Santi Padri"79, dalla "Regola di Macario"80, dalle "Regole di Paolo e Stefano" $"$, dalla "Regola del Maestro" ${ }^{82}$ Nelle "Regole" di sant'Aureliano può essere anche scorto un influsso di san Benedetto di Norcia ${ }^{83}$.

Concludendo, possiamo affermare che la "Regola" femminile di san Cesario d'Arles è antecedente rispetto alla Regula monasteriorum di san Benedetto da Norcia. Fra le due "Regole", così come le conosciamo oggi, c'è senz'altro qualche somiglianza, al punto che più di uno studioso moderno ha pensato ad una vicendevole dipendenza. La questione, però, rimane aperta. A dir il vero, a nostro avviso, è difficile che la Regula monasteriorum benedettina possa annoverarsi tra le fonti degli Statuta virginum, perché quando san Cesario si accinse alla composizione di questo documento giuridico-spirituale-monastico, la "Regola" di san Benedetto non era stata ancora stilata e, d'altra parte, le posteriori modifiche ed aggiunte, fatte da san Cesario agli Statuta virginum, sono dovute alla lezione dell'esperienza. È altresì poco probabile che, prima del 523, san Benedetto abbia letto la "Regola" di san Cesario. Le somiglianze, quindi, che intercorrono fra le due "Regole", sono dovute alla comunanza delle fonti e alla stessa prassi monastica ormai diffusa in Occidente.

79 Pubblicata in PG 34, 971-978 e PL 103, 435-442; ed. A. de Vogüé, SCh 297, Paris 1982, $274-$ 282; ed. S. Pricoco, in: La Regola di San Benedetto e le Regole dei Santi Padri, Milano 1995, 25-35. Cf. anche J. Neufville, Regula Patrum secunda, DIP VII 1590-1591; A. de Vogüé, La règle de Vigile signalé par Gennade. Essai d'identification, RBen 89 (1979) 217-229; idem, Histoire littéraire du mouvement monastique dans l'antiquité, Première partie: Le monachisme latin, t. 7: L'essor de la littérature lérinienne et les écrits contemporains (410-500), Paris 2003, 56-77.

${ }^{80}$ Pubblicata in PG 34, 967-970; PL 103, 447-452; ed. H. Styblo: Die Regula Macharii, WS 76 (1963) 124-158; ed. A. de Vogüé, SCh 297, 372-388; ed. Pricoco in: La Regola di San Benedetto e le Regole dei Santi Padri, p. 37-57. Cf. anche F. Massai, Les antécédents de Cluny. Règle du Maître à Moutiers-Saint-Jean, in: À Cluny. Congrès scientifique. Fêtes et Cérémonies liturgiques en l'honneur des saints Abbés Odon et Odilon, 9-11 juillet 1949, Dijon 1950, 192-202; A.C. Vega, En torno a la herencia literaria de Juan de Biclaro, "Boletín de la Real Academia de Historia" 164 (1969) 13-74; A. de Vogüé, Trithème, La Règle de Macaire et l'héritage littéraire de Jean de Biclar, SE 23 (1978-1979) 217-224; idem, La „Vita Pachomii Iunioris” (BHL 6411). Ses rapports avec la Règle de Macaire, Benoît d'Aniane et Fructueux de Braga, SM 20 (1979) 535-553; idem, Regula Macarii, DIP VII 1580-1582; idem, Histoire littéraire du mouvement monastique dans l'antiquité, t. 7, p. 399-439.

${ }^{81}$ Pubblicata in PL 66, 949-958; ed. J.E.M. Vilanova: Regula Pauli et Stephani, Scripta et documenta 11, Montserrat 1959. Cf. anche U. Berlière, La Règle des saints Étiennes et Paul, in: Mélanges Paul Thomas. Recueil de mémoires concernant la philologie classique dédié à Paul Thomas, Bruges 1930, 39-53; J.E.M. Vilanova, Regula Pauli et Stephani, DIP VII 1592-1594; A. de Vogüé, Histoire littéraire du mouvement monastique dans l'antiquité, Première partie: Le monachisme latin, t. 9: De Césaire d'Arles à Grégoire de Tours (525-590), Paris 2005, 183-198.

${ }^{82}$ Pubblicata in SCh 105-106, Paris 1964.

${ }^{83}$ Cf. Gobry, Storia del monachesimo, I, p. 650. 


\section{SAINT CAESARIUS OF ARLES AND HIS FOLLOWERS AND WOMEN MONASTICISM}

\section{(Summary)}

The article presents the Western Women Monasticism in Gaul spread by Saint Caesarius of Arles and his followers. In 506 Saint Caesarius of Arles undertook the building of the Women Monastery of Saint John, which was finalized in 512. The first Abbess of the monastery was Saint Caesarius' sister, Saint Caesaria.

Saint Caesarius wrote the Rule for the monastery in 534. After Saint Caesaria, another Caesaria became Abbess and the monastery counted up to 200 nuns in those years. To Abbess Caesaria, Saint Liliola and Rusticula succeeded.

The influence of Saint Caesarius on Monasticism in Gaul was relevant, thought the Rule had a greater impact in Provence.

The history of the monastery of Saint Andrew at Villeneuve, near Avinione, is not widely attested: one chronicle reports that, in 586, a woman named Caesaria died in the monastery. Even fewer historical sources concern other women monasteries in Gaul. According to tradition, also the monastery of Saint Peter du Puy in Orange was founded by Saint Caesarius.

In the VI century the Rules of Saint Aurelianus of Arles, Regula ad monachos and Regula ad virgines were written as well. Saint Aurelianus of Arles founded two monasteries in Arles: a women monastery (Saint Mary's) and a men monastery (Saints Peter and Paul's).

\section{ŚW. CEZARY Z ARLES I JEGO KONTYNUATORZY A MONASTYCYZM ŻEŃSKI}

\section{(Streszczenie)}

Artykuł omawia łaciński monastycym żeński w Galii krzewiony przez św. Cezarego z Arles i jego kontynuatorów. W 506 r. Cezary rozpoczął budowę klasztoru żeńskiego św. Jana, która została ukończona w 512 r. Pierwszą jego ksienią została siostra św. Cezarego - św. Cezaria. Do klasztoru wstapiło wiele dziewic i wdów. Cezary napisał dla nich regułę w 534 r. Następczynią św. Cezarii została inna Cezaria, zwana Młodszą. Mniszek w klasztorze było wówczas aż dwieście. Następną ksienią została św. Liliola, a później św. Rustykuła.

Przed śmiercią Cezary chciał uczestniczyć w liturgii mniszek w klasztorze św. Jana i skierował do wspólnoty zachętę, by pozostała wierna regule. Wpływ Cezarego na monastycyzm galijski był wielki, choć „Reguła” odegrała większą rolę jedynie w Prowansji.

Nie znamy dokładnie dziejów klasztoru św. Andrzeja z Villeneuve koło Awinionu. Jedna z kronik mówi, iż w 586 r. zmarła w nim niejaka św. Cezaria. Jeśli chodzi o inne klasztory żeńskie w Galii, to mamy na ich temat jeszcze bardziej skappe wiadomości. Zachował się list Cezarego wysłany do pewnej ksieni, 
z którego można wnosić, iż była ona jego duchową córką i kierowała założoną przez niego wspólnotą mniszek. Według tradycji także klasztor św. Piotra du Puy w Orange został założony przez św. Cezarego na górze Eutropiusza.

W VI w. powstały także „Reguły” św. Aureliana: Regula ad monachos i Regula ad virgines. Założył on w Arles dwa klasztory: żeński św. Marii i męski świętych Piotra i Pawła.

Key words: Saint Caesarius of Arles, Saint Aurelianus of Arles, Monasticism, Woman.

Parole chiave: san Cesario d'Arles, sant'Aureliano di Arles, monachesimo, donna.

Słowa kluczowe: św. Cezary z Arles, św. Aurelian z Arles, monastycyzm, kobieta.

\section{BIBLIOGRAFIA}

\section{Fonti}

Agapitus, Epistula [ad sanctum Caesarium Arelatensem] (18 VII 535), ed. C. de Clercq, CCL 148A, Turnholti 1963, 96-97.

Augustinus Hipponensis, Regula, PL 32, 1377-1384; ed. A. Goldbacher, CSEL 57, Vindobonae - Lipsiae 1911, 356-371; Schröder P., Die Augustinerchorherrenregel: Entstehung, kritischer Text und Einführung der Regel, "Archiv für Urkundenforschung" 9 (1926) 271-306; Bruyne D. de, La première Règle de saint Benoît, RBen 42 (1930) 316-342; Vega A.C., La Regla de San Agustín. Edición crítica, precedida de un estudio sobre la misma y los códices de El Escorial, El Escorial 1933; Arbesmann R. - Huempfner W., Jordani de Saxonia liber vitae fratrum, New York 1943, 491-504; Verheijen L., La Règle de saint Augustin, Collection des Études Augustiniennes, Paris 1967 (Obiurgatio: 105-107; Ordo monasterii: 148-152; Praeceptum: 417-437).

Aurelianus Arelatensis, Regula ad monachos, PL 68, 385-398; ed. A. Schmidt, Zur Komposition der Mönchsregel des heiligen Aurelian von Arles, SMon 17 (1975) 237-256 (edizione critica) e SMon 18 (1976) 17-54.

Aurelianus Arelatensis, Regula ad virgines, PL 68, 399-406.

Bonifacius II, Epistula (25 I 531), ed. C. de Clercq, CCL 148A, Turnholti 1963, 66-69.

Caesarius Arelatensis, Ad sanctimoniales epistulae, PL 67, 1128C - 1135C; ed. G. Morin in: Sancti Caesarii episcopi Arelatensis, Opera omnia, II, Maredsous 1942, 134-144; éd. A. de Vogüé - J. Courreau in: Césaire d'Arles, Oeuvres Monastiques, SCh 345, Paris 1988, 294-336.

Caesarius Arelatensis, Epistula ad Bonifacium II, ed. C. de Clercq, CCL 148A, Turnholti 1963, 69.

Caesarius Arelatensis, Statuta sanctarum virginum, PL 67, 1105-1120; ed. G. Morin in: Sancti Caesarii episcopi Arelatensis, Opera omnia, I, Maredsous 1937, 100-127; éd. A. de Vogüé - J. Courreau in: Césaire d'Arles, Oeuvres Monastiques, SCh 345, Paris $1988,170-272$.

Caesarius Arelatensis [?; Teridius ?], Epistula hortatoria ad virginem Deo dicatam, PL 67, 1135-1138; éd. A. de Vogüé - J. Courreau in: Césaire d'Arles, Oeuvres Monastiques, SCh 345, Paris 1988, 418-438. 
Concilium Arelatense (6 VI 524), ed. C. de Clercq, CCL 148A, Turnholti 1963, 42-46.

Concilium Carpentoratense (6 XI 527), ed. C. de Clercq, CCL 148A, Turnholti 1963, 47-52.

Concilium Arausicanum (3 VII 529), ed. C. de Clercq, CCL 148A, Turnholti 1963, 53-76. Concilium Massiliense (26 V 533), ed. C. de Clercq, CCL 148A, Turnholti 1963, 84-97.

Concilium Vasense (5 XI 529), ed. C. de Clercq, CCL 148A, Turnholti 1963, 77-81.

Epistolae Romanorum Pontificum, ed. A. Thiel, I, Braunsberg 1868.

Felx IV, Epistula (3 II 528), ed. C. de Clercq, CCL 148A, Turnholti 1963, 51-52.

IoAnnes Cassianus, Conlationes patrum, PL 49, 477-1321; ed. M. Petschenig, CSEL 13a,

Wien 2004; ed. E. Pichery, t. 1: I-VII, SCh 42, Paris 1955; t. 2: VIII-XVII, SCh 54,

Paris 1958; t. 3: XVIII-XXIV, SCh 64, Paris 1959.

Ioannes CAssianus, De institutis coenobiorum, PL 49, 43-476; ed. M. Petschenig, CSEL

17, Pragae - Vindobonae - Lipsiae 1888, 3-231; ed. J.-C. Guy, SCh 109, Paris 1965.

IoAnNes II, Epistula [ad clerum Reiensem], ed. C. de Clercq, CCL 148A, Turnholti 1963, 86-86.

IoAnNes II, Epistula [ad episcopos Galliae], ed. C. de Clercq, CCL 148A, Turnholti 1963, 86. IOANNES II, Epistula [ad sanctum Caesarium Arelatensem] (7 IV 534), ed. C. de Clercq, CCL 148A, Turnholti 1963, 87-96.

Regula Macarii, PG 34, 967-970; PL 103, 447-452; ed. H. Styblo: Die Regula Macharii, WS 76 (1963) 124-158; ed. A. de Vogüé, SCh 297, Paris 1982, 372-388; ed. S. Pricoco, in: La Regola di San Benedetto e le Regole dei Santi Padri, Milano 1995, 37-57.

Regula Magistri, ed. A. de Vogüé, SCh 105-106, Paris 1964.

Regula Pauli et Stephani, PL 66, 949-958; ed. J.E.M. Vilanova: Regula Pauli et Stephani, Scripta et documenta 11, Montserrat 1959.

Regula Sanctorum Patrum Secunda, PG 34, 971-978 e PL 103, 435-442; ed. A. de Vogüé, SCh 297, Paris 1982, 274-282; ed. S. Pricoco, in: La Regola di San Benedetto e le Regole dei Santi Padri, Milano 1995, 25-35.

Vita Sancti Caesarii Episcopi Arelatensis, ASanc (mensis Augusti) VI 50-83; ed. B. Krusch, Vitae Caesarii episcopi Arelatensis libri duo, MGH Scriptores rerum Merovingicarum III, Hannoverae 1896, 433-501; ed. E. Bona, Vita sancti Cesarii Episcopi Arelatensis, Supplementi di Lexis 16, Amsterdam 2002; ed. M.-J. Delage - M. Heijmans, Vie de Césaire d'Arles, SCh 536, Paris 2010.

Studi

Altamira R., La Spagna sotto i visigoti, in Storia del mondo medievale, I, Milano 1999, 743-779.

Amory P., People and identity in Ostrogothic Italy, 489-554, Cambridge 1997.

BerLière U., La Règle des saints Étiennes et Paul, in: Mélanges Paul Thomas. Recueil de mémoires concernant la philologie classique dédié à Paul Thomas, Bruges 1930, $39-53$.

Degórski B., Il primissimo monachesimo della Gallia, DPa 15 (2006) 5-66.

Degórski B., Il monachesimo latino nel Mediterraneo (secc. IV-VI): protagonisti e legislazione, Lublin 2006.

EnssLin W., Theoderich der Grosse, München 1947.

Giovanditto A., Teodorico e i suoi Goti in Italia (454-526), Milano 1998.

Gobry I., Storia del monachesimo, I-II, Roma 1991.

Hen Y., Scrittori della Gallia, in: Quasten IV 249-365.

KasPer C., Cipriano di Tolone, in: Dizionario di letteratura cristiana antica, ed. S. Döpp - W. Geerlings - C. Noce, Città del Vaticano - Roma 2006, 190. 
Lамвот C., La Règle de S. Augustin et S. Césaire, RBen 41 (1929) 331-341.

Lамвот C., Le prototype des monastères cloîtrés de femmes: l'Abbaye Saint-Jean d'Arles (VIe siècle), "Revue Liturgique et Monastique" 23 (1938) 169-174.

Lamma P., Teodorico, La Scuola Editrice, Brescia 1951.

Limmer J., Konzilien und Synoden im spätantiken Gallien von 314 bis 696 nach Christi Geburt, Teil 1: Chronologische Darstellung, Wissenschaft und Religion 10, Frankfurt am Main 2004.

Massai F., Les antécédents de Cluny. Règle du Maître à Moutiers-Saint-Jean, in: À Cluny. Congrès scientifique. Fêtes et Cérémonies liturgiques en l'honneur des saints Abbés Odon et Odilon, 9-11 juillet 1949, Dijon 1950, 192-202.

Moorhead J., Theoderic in Italy, Oxford 1992.

Morin G., Problèmes relatifs à la Règle de S. Césaire d'Arles pour les moniales, RBen 44 (1932) 5-20.

Morin G., Le prêtre arlésien Teridius, propagateur des Régles de S. Céaire d'Arles, RSR 27 (1938) 257-263.

Neufville J., Regula Patrum secunda, DIP VII 1590-1591.

Pfister CH., La Gallia sotto i Franchi merovingi, vicende storiche, in: Storia del mondo medievale, I, Milano 1999, 688-711.

Pfister CH., La Gallia sotto i Franchi merovingi, istituzioni, in: Storia del mondo medievale, I, Milano 1999, 712-742.

Pilvousek J. - Springer K.B., Caesarius von Arles und die Klostergründung der heiligen Radegunde, in: Radegunde - ein Frauenschicksal zwischen Mord und Askese: eine Ausstellung, hrsg. H. Eidam - G. Noll, Erfurt 2006, 79-95.

PiŁAt J., Św. Cezary z Arles. Bibliografia - aktualny stan badań, in: Oblicza doskonałości chrześcijańskiej, red. M. Chmielewski, Lublin 1996, 206-218.

SAItTA B., La "civilitas" di Teodorico: rigore amministrativo, "tolleranza” religiosa e recupero dell'antico nell'Italia ostrogota, Roma 1999.

Sardella T., Cesario di Arles, in: Quasten III 290-295.

SAXER V., Cipriano di Tolone, DPAC I 683.

SAXER V., Cipriano di Tolone, NDPAC I 1036-1037.

Seinac L. DE, Remarques sur l'utilisation par Césaire d'Arles de la "Regula Augustini, "Regulae Benedicti Studia. Annuarium internationale" 1 (1972) 287-291.

Seinac L. DE, L'Utilisation par s. Césaire d'Arles de la Règle de s. Augustin. Étude de terminologie et doctrine monastique, Studia Anselmiana 62, Roma 1974.

Spinelli M., Introduzione, in: Giuliano Pomerio, La vita contemplativa, Collana di Testi Patristici 64, Roma 1987, 7-41.

Starowieyski M., Prawodawstwo św. Cezarego z Arles, in: Zachodnie Reguly monastyczne, ŹM 50, Kraków - Tyniec 2013², 387-403.

Terraneo G., Saggio bibliografico su Cesario di Arles, SC 91 (1963) 232-294.

Thiellet C., La Règle de saint Césaire d'Arles et les fondations monastiques féminines, in: Pratique et sacré dans les espaces monastiques au Moyen-Âge et à l'époque moderne. Actes du colloque de Liessies- Maubeuge (26-28 septembre 1997), éd. R.-H. Bautier - Ph. Racinet, Amiens 1998, 23-33.

Tibiletti C., La teologia della grazia in Giuliano Pomerio, “Augustinianum” 25 (1985) 489-506.

Vega A.C., En torno a la herencia literaria de Juan de Biclaro, "Boletín de la Real Academia de Historia" 164 (1969) 13-74.

Vilanova J.E.M., Regula Pauli et Stephani, DIP VII 1592-1594. 
VogüÉ A. De, La Règle de Saint Césaire d'Arles pour les moines: un résumé de sa Règle pour les moniales, RAM 47 (1971) 369-406.

VogüÉ A. DE, Trithème, La Règle de Macaire et l'héritage littéraire de Jean de Biclar, SE 23 (1978-1979) 217-224.

VogüÉ A. DE, La règle de Vigile signalé par Gennade. Essai d'identification, RBen 89 (1979) 217-229.

VogüÉ A. DE, La "Vita Pachomii Iunioris" (BHL 6411). Ses rapports avec la Règle de Macaire, Benoît d'Aniane et Fructueux de Braga, SM 20 (1979) 535-553.

VogüÉ A. DE, Une interpolation inspirée de Cassien dans un texte monastique Césaire d'Arles, SMon 25 (1983) 217-221.

VogüÉ A. De, Regula(e) Aureliani, DIP VII 1604-1607.

VogüÉ A. DE, Regula Macarii, DIP VII 1580-1582.

VogÜÉ A. DE, Histoire littéraire du mouvement monastique dans l'antiquité, Première partie: Le monachisme latin, t. 7: L'essor de la littérature lérinienne et les écrits contemporains (410-500), Paris 2003.

VogüÉ A. DE, Histoire littéraire du mouvement monastique dans l'antiquité, Première partie: Le monachisme latin, t. 9: De Césaire d'Arles à Grégoire de Tours (525-590), Paris 2005. 